\title{
RESULTADOS Y PROPUESTAS DE UN SERVICIO DE ORIENTACIÓN UNIVERSITARIA*
}

\author{
GUIDANCE SERVICE IN UNIVERSITIES: RESULTS AND PROPOSALS FROM
}

\author{
Benito del Rincón Igea** y Agustín Bayot Mestre*** \\ Universidad de Castilla-La Mancha
}

\section{RESUMEN}

En esta aportación nos proponemos justificar con algunas investigaciones realizadas en el contexto de la Universidad de Castilla-La Mancha (Campus de Cuenca) que, en el marco de nuestras estructuras universitarias, es necesario crear órganos de orientación y apoyo a los estudiantes como condición para que éstos consigan la formación que el mundo laboral y la sociedad en general les exigen.

Los resultados obtenidos indican que el 98\% de los participantes consideran necesaria la existencia de un servicio de estas características, el 30\% del alumnado manifiesta no tener información acerca de la proyección laboral de su carrera y el $94,60 \%$ señala que la Universidad no le ha facilitado ninguna ayuda posterior a la finalización de los estudios para obtener un puesto de trabajo. Además, aportamos referentes que pueden ser de utilidad para organizar uno de estos servicios.

Palabras clave: Servicio de orientación universitaria, inserción laboral, prevención de conductas de riesgo.

\section{ABSTRACT}

On this contribution, we will be able to justify, having carried out recent researches in the University of Castilla La Mancha (Campus of Cuenca), the necessity of the establishment of guidance and su-

* El proyecto fue aprobado, y subvencionado, por la Dirección General de Enseñanza Universitaria e Investigación, de la Junta de Comunidades de Castilla-La Mancha.

Aprovechamos para mostrar nuestro agradecimiento a D. Ángel García (Director de la Residencia Bartolomé de Cossio), a D a Soledad Argudo, D. Carlos F. Racionero y a Da Susana González por su colaboración en este estudio.

** Benito del Rincón Igea: Profesor Titular de la Facultad de CC de la Educación y Humanidades de la UCLM. Posee una amplia experiencia en formación inicial y permanente del profesorado y algunos de sus ámbitos de estudio se relacionan con la atención a la diversidad, el trabajo psicopedagógico y la orientación universitaria. Sobre estos temas ha publicado varias obras y artículos de revista. B.Rincon@uclm.es

*** Agustín Bayot Mestre: Profesor Titular del área de Métodos de Investigación y Diagnóstico en Educación de la Universidad de Castilla-La Mancha (UCLM). Entre sus líneas de trabajo se encuentran metodología de investigación, validación de instrumentos de medida y orientación universitaria. agustin.bayot@uclm.es 
pport services for students as a condition to ensure they obtain the best possible university education available in order to enable the students to overcome the difficulties they can find when they conclude their university studies.

The collected information show that $98 \%$ of the participants consider that it is necessary the establishment of such kind of services, $30 \%$ of the students declare having no information about what kind of post-graduated studies or jobs they can apply for and $94,60 \%$ assure that they have received no support from University institution when looking for a job. Furthermore, we add strategies in order to develop these kind of services.

Key words: University guidance service, employment insertion, risk behavior prevention.

\section{Introducción}

Es evidente que, desde hace ya algunas décadas, la institución universitaria está siendo sometida a un proceso de discusión que, sin duda, ha generado y va a generar cambios sustanciales. Entre los puntos de debate, podemos encontrar las funciones que desempeña, o debe desempeñar, en la sociedad actual. ¿Qué fines persigue la institución de educación superior por excelencia? Es indiscutible su papel fundamental en la formación de profesionales y en la investigación, pero también adquiere cada vez más sentido una meta de carácter global: la formación humana de los estudiantes. De hecho, ésta se encuentra en la base de todo profesional o investigador.

En este sentido, la visión de Vázquez, Colom y Sarramona (1998: 56) puede ser equilibrada cuando expresan una concepción de la universidad cercana a la formación de las personas, a la transmisión del conocimiento y a la preparación de profesionales.

Seguramente, una de las adaptaciones más urgentes que la universidad debe alcanzar consiste, precisamente, en la formación de competencias básicas que los estudiantes puedan transferir con relativa facilidad de unos campos de conocimiento a otros. Martínez Aldanondo (2002: 32) destaca, entre otras, las siguientes:
a) trabajo en equipo;
b) creatividad e innovación:
c) inteligencia emocional;
d) capacidad de aprender y desaprender;
e) hablar en público y
f) resolución de problemas.

A propósito de la armonización de la Universidad española con los principios del Espacio Europeo de Educación Superior (EEES), se han elaborado los Libros Blancos de las distintas titulaciones futuras y, todos ellos, abundan en la importancia de las competencias enumeradas $^{1}$. La mayoría de ellas guardan estrecha relación con el perfil humano de los individuos. Pero estas capacidades, cada vez más demandadas en el acceso al mundo empresarial, se descuidan generalmente ${ }^{2}$.

1. http://www.aneca.es/modal_eval/conver_docs_titulos.html

2. Según el informe sobre el Plan Nacional de Calidad en las Universidades (convocatoria de 1996), predomina la clase magistral (Consejo de Universidades, 1997). 
En los últimos años han proliferado alternativas y propuestas para responder a las necesidades que venimos comentando; fundamentalmente los Servicios de Orientación Universitaria que, de hecho, existen en casi todas las universidades, aunque todavía no es una realidad en la de Castilla-La Mancha. En general, se trata de acciones organizadas desde una perspectiva de "servicios" que van dirigidos a quienes necesitan alguna información o ayu$\mathrm{da}$, descontextualizados y poco integradas en el conjunto de la universidad, con escasos recursos y sin personal especializado (Vieira y Vidal, 2006: 91).

En relación con todo lo expuesto, en este trabajo daremos cuenta de los resultados obtenidos en varios estudios realizados en la Universidad de Castilla-La Mancha con muestras amplias de alumnado universitario y titulado.

\section{Descripción de una experiencia}

Durante el curso 1999-2000 se desarrolló un proyecto de servicio de orientación universitaria, en el seno de la Residencia Universitaria "Bartolomé Cossío" de Cuenca, bajo nuestra coordinación y asesoramiento. Esta acción pionera en el contexto universitario de Castilla-La Mancha respondería, inicialmente, a las necesidades de los estudiantes de dicha Residencia Universitaria, aunque con intención, a medio plazo, de atender a todo el Campus de la ciudad ${ }^{3}$. La experiencia se inició con la perspectiva de incorporar el servicio a la Universidad, aunque distintos avatares de política educativa, derivados en parte de los nuevos marcos legales, han hecho inviable todavía esta generalización ${ }^{4}$.

La justificación del proyecto podría situarse en las razones generales por las que la universidad debe contar con servicios de orientación, así como por la pertinencia de un servicio que cumpliera una labor de apoyo, no incluida en las tareas del Servicio de Información al Estudiante (SIE) ya existente en el campus ${ }^{5}$. Pretendíamos alcanzar los siguientes objetivos (Del Rincón, Bayot, García y Argudo, 2000):

a) situar al estudiante en el entorno universitario general y en el propio de su facultad o escuela universitaria donde inicie sus estudios;

b) ayudar a conocer los itinerarios formativos de grado y postgrado de las distintas titulaciones;

c) apoyar los procesos de aprendizaje en la universidad, y

d) orientar la toma de decisiones en el mundo laboral.

La experiencia fue aprobada por la Dirección General de Enseñanza Universitaria e Investigación del Gobierno Autonómico ${ }^{6}$ y se dotó con becas de estancia en la Residencia que aprovecharían dos estudiantes de Psicopedagogía en calidad de colaboradoras del proyecto 7 . Tal

3. La UCLM se distribuye por toda la Región, excepto Guadalajara, en varios Campus: Albacete, Ciudad Real, Cuenca, Toledo, Talavera y Almadén.

4. Desde el Vicerrectorado de Alumnos se está impulsando también la creación, en cada campus, de un Centro de Información para el Empleo (CIPE).

5. Una información más detallada sobre esta aportación puede verse en Del Rincón y otros (2000).

6. Escrito del 14 de octubre de 1999.

7. El equipo del servicio se constituye de esta forma: Dr. Benito del Rincón y Dr. Agustín Bayot (asesores). Ángel García, Soledad Argudo, Carlos F. Racionero y Susana González. 
como se especificaba en la programación inicial, las actuaciones a desarrollar se distribuyeron en los programas o apartados que enumeramos a continuación (Del Rincón et al., 2000):

a) acogida e incorporación al contexto universitario: ubicación y conocimiento del entorno; encuentro general y presentación del SOUC;

b) orientación personal: prevención y desarrollo;

c) orientación académica: el estudio en la universidad, estrategias y planificación del tiempo; base de datos con las características de las asignaturas de las distintas carreras; planes de estudios, itinerarios y optatividad y

d) orientación profesional: recogida de información de los egresados en promociones anteriores de algunas carreras del Campus de Cuenca.

De forma más precisa, a continuación damos cuenta de las actividades llevadas a cabo:

a) Acogida e incorporación al contexto universitario.

b) Orientación personal: prevención y desarrollo. Jornada sobre "Hábitos de estudio y relajación" durante el período de exámenes.

c) Orientación académica. Técnicas de estudio y organización del tiempo en la universidad y

d) Orientación profesional. El SOUC considera fundamental poner en marcha un programa de apoyo a la búsqueda de empleo de los graduados universitarios.

La mitad de los participantes afirmaron no conocer el Servicio de Información Universitaria (SIE) y, de los que lo conocían, señalaban que no ofrece suficiente información. Respecto de la existencia de un Servicio de Orientación Universitaria, casi la totalidad de los participantes están de acuerdo con su existencia $(95,1 \%)$. Consideraban muy adecuada la prestación de servicios referentes a ayudas para la mejora del aprovechamiento y el rendimiento académico general y ayudas a su situación emotiva personal, así como la implantación de programas de educación para la salud (SIDA, información sexual, drogodependencias, etc.). Sobre estos resultados, puede encontrarse más información en Bayot A., "et als" (2000).

\section{Método}

Nos referimos a dos estudios realizados acerca de las funciones que debe desempeñar un servicio de orientación, por una parte, así como determinar la situación en la que se encuentra una muestra de egresado después de haber finalizado sus estudios, por otra.

\section{Estudio I}

\section{a) Objetivos}

El objetivo fundamental radica en analizar la opinión de los estudiantes de la Universidad de Castilla-La Mancha alojados en las Residencias Universitarias de la Consejería de Educación (octubre, 2000), sobre la función que puede cumplir un servicio de orientación universitaria. 


\section{b) Procedimiento y análisis estadísticos}

Para tal fin, se elaboró una encuesta en la que se planteaban, entre otras cuestiones, la necesidad de un servicio de orientación, las funciones que debe cumplir, etc.

Desde la Residencia Universitaria Bartolomé de Cossío (Campus de Cuenca), se remitieron 1918 encuestas a todas las residencias universitarias de la UCLM, consideradas finalmente válidas 837 .

Con relación a las pruebas estadísticas realizadas, se aplicaron análisis descriptivos y pruebas de diferencias por medio de análisis de varianza. En aquellos casos en los que se han obtenido diferencias significativas, se ha aplicado la prueba de comparación de medias a posteriori de Tukey. Para evitar el error tipo I, se ha realizado la siguiente corrección: $\alpha=0.05 / 14=0,003$.

\section{c) Descripción de la muestra}

La encuesta fue cumplimentada por 837 participantes $(\mathrm{N}=837)$ sobre una población máxima de 1918 (la muestra supone, aproximadamente, el 42,5\% del total de residentes universitarios y el $2,54 \%$ de todos los estudiantes de la UCLM), con un promedio de edad de 20,11 años $(X=20,11 ; \mathrm{Sx}=1,72)$. El $45,70 \%$ son hombres y el $54,30 \%$ mujeres. El $14,7 \%$ estudia una carrera experimental (formado por 7 carreras), el 17,2\% humanidades (4 carreras), el 32,6\% técnicas (11 carreras) y el 35,5\% sociales (11 carreras). El 83,6\% está matriculado en la carrera que eligió en primer lugar.

\section{c) Descripción del Instrumento}

Con el fin de obtener la información relativa a las opiniones de los estudiantes sobre la existencia de un servicio de información en la universidad, se elaboró el "Cuestionario de Información Universitaria", formado por 36 items de respuesta tipo Likert, cuya cumplimentación pudo realizarse al inicio del curso 1999/2000, entre los alumnos universitarios de primer curso, alojados en residencias universitarias de la Junta de Comunidades de Castilla-La Mancha (Campus de Cuenca). Sobre un total posible de 230 participantes, 103 cumplimentaron la encuesta de forma válida.

\section{Resultados}

808 participantes $(98,10 \%)$ opinan que debe existir un servicio de estas características. Entre este porcentaje, el $97,90 \%$ contesta que este servicio tiene que ser para todos los universitarios y el $2,10 \%$ sólo para los que se encuentran en una residencia.

Además, tuvieron que valorar, entre uno y cinco, una serie de actividades que puede llevar a cabo un servicio de orientación (tabla 1). Como se puede apreciar en dicha tabla, en todos los casos se ha obtenido un valor promedio elevado, excepto en "relajación" y en "sexualidad y hábitos saludables". A partir del ANOVA aplicado, únicamente existen diferencias significativas en la "preparación de las oposiciones" $[\mathrm{F}(3,679)=6,16 ; \mathrm{p}<0,0005]$ y en "atención psicopedagógica individual" $[\mathrm{F}(3,668)=3,89 ; \mathrm{p}<0,0005]$, entre las dife- 
TABLA 1: Pruebas de diferencias

\begin{tabular}{|l|c|c|c|c|c|c|c|c|c|c|}
\hline & \multicolumn{2}{|c|}{ Experimentales } & \multicolumn{2}{c}{ Humanidades } & \multicolumn{2}{|c|}{ Técnicas } & \multicolumn{2}{c|}{ Sociales } & \multirow{2}{*}{$\mathbf{F}$} & $\mathbf{p}$ \\
\hline \multicolumn{1}{|c|}{ Variables } & $\mathbf{X}$ & $\mathbf{S x}$ & $\mathbf{X}$ & $\mathbf{S x}$ & $\mathbf{X}$ & $\mathbf{S x}$ & $\mathbf{X}$ & $\mathbf{S x}$ & & \\
\hline Técnicas de estudio & 3,80 & 1,35 & 3,40 & 1,55 & 3,61 & 1,38 & 3,61 & 1,40 & 1,53 & 0,20 \\
\hline Superación exámenes & 3,68 & 1,35 & 3,59 & 1,38 & 3,77 & 1,37 & 3,86 & 1,24 & 1,21 & 0,30 \\
\hline Buscar trabajo & 4,58 & 0,91 & 4,33 & 1,09 & 4,53 & 0,86 & 4,47 & 1,03 & 1,43 & 0,23 \\
\hline Hablar en público & 4,03 & 1,11 & 4,00 & 1,25 & 3,86 & 1,14 & 4,00 & 1,18 & 0,82 & 0,48 \\
\hline Tomar decisiones & 3,64 & 1,25 & 3,47 & 1,40 & 3,53 & 1,28 & 3,66 & 1,22 & 0,79 & 0,49 \\
\hline Técnicas de relajación & 3,18 & 1,32 & 3,07 & 1,51 & 2,81 & 1,41 & 2,96 & 1,35 & 1,87 & 0,13 \\
\hline Salidas laborales & 4,39 & 0,96 & 4,26 & 1,12 & 4,23 & 1,07 & 4,25 & 1,13 & 0,56 & 0,64 \\
\hline $\begin{array}{l}\text { Preparación } \\
\text { oposiciones }\end{array}$ & 4,17 & 0,95 & 4,23 & 1,08 & 3,73 & 1,22 & 4,10 & 1,25 & 6,51 & 0,00 \\
\hline $\begin{array}{l}\text { Sexualidad/hábitos } \\
\text { saludables }\end{array}$ & 3,17 & 1,30 & 3,07 & 1,29 & 3,06 & 1,44 & 3,02 & 1,44 & 0,26 & 0,85 \\
\hline Mejoras profesionales & 4,10 & 1,14 & 4,03 & 1,07 & 4,12 & 1,06 & 4,05 & 1,05 & 0,25 & 0,85 \\
\hline $\begin{array}{l}\text { Atención } \\
\text { psicopedagógica }\end{array}$ & 3,63 & 1,13 & 3,72 & 1,24 & 3,28 & 1,34 & 3,38 & 1,31 & 3,88 & 0,00 \\
\hline $\begin{array}{l}\text { Información mercado } \\
\text { laboral }\end{array}$ & 4,22 & 1,02 & 4,13 & 1,13 & 4,11 & 1,17 & 4,14 & 1,08 & 0,21 & 0,88 \\
\hline Becas/ayudas & 4,56 & 0,79 & 4,41 & 0,98 & 4,50 & 0,87 & 4,37 & 1,01 & 1,30 & 0,27 \\
\hline $\begin{array}{l}\text { Transición } \\
\text { universidad/trabajo }\end{array}$ & 4,48 & 0,93 & 4,38 & 1,04 & 4,30 & 0,94 & 4,35 & 1,02 & 0,80 & 0,49 \\
\hline
\end{tabular}

$\mathrm{X}=$ Media; $\mathrm{Sx}=$ Desviación típica; $\mathrm{F}=$ Prueba de contraste de hipótesis $\mathrm{F}$ Ratio; $\mathrm{p}=$ probabilidad; Prueba de Tukey: $\mathrm{A}=$ Experimentales-Técnicas; $\mathrm{B}=$ Humanidades-Técnicas; $\mathrm{C}=$ Técnicas-Sociales.

rentes carreras cursadas (experimentales, humanísticas, técnicas y sociales) (tabla 1). Fundamentalmente, las diferencias se encuentran a favor de las carreras de humanidades.

\section{Estudio II}

\section{a) Objetivos}

Analizar las dinámicas y tendencias generales de formación y empleo desarrolladas por una muestra de profesionales que se formaron en la UCLM y, concretamente, en el Campus de Cuenca. 


\section{b) Procedimiento y análisis estadísticos}

Para tal finalidad, se elaboró el "Cuestionario de egresados de la UCLM", formado por 16 preguntas de respuesta múltiple. Recoge información sobre una serie de indicadores acerca de ámbitos como los siguientes:

a) orientación recibida con respecto a salidas laborales;

b) formación complementaria al término de los estudios: postgrados, master, etc.;

c) relación entre los estudios realizados y el trabajo actual y

d) itinerario laboral y situación actual.

La intención era recabar información para orientar con más referentes a los estudiantes que finalizarán sus estudios en un futuro próximo. El cuestionario se remitió en febrero de 2000 a los alumnos que habían finalizado sus estudios durante los cursos 1995 y 1997, convertidos ya en profesionales u ocupados en la búsqueda de empleo. Para cada una de las variables contempladas, se han aplicado análisis descriptivos.

\section{c) Descripción de la muestra}

Se seleccionaron a los egresados que habían cursado las carreras de Bellas Artes, Trabajo Social y Enfermería. Está formada por 9 hombres $(11,80 \%)$ y 67 mujeres $(88,20 \%)$ $(\mathrm{N}=76)$, con una media de edad de 26,16 años en el momento actual $(\mathrm{X}=26,16$; $\mathrm{Sx}=3,06)$. Con relación a los estudios realizados, 28 cursó la carrera de Trabajo Social $(36,80 \%), 13$ Bellas Artes $(17,10 \%)$ y 35 Enfermería (46,10\%). En cuanto a su lugar de procedencia, 36 participantes $(48,00 \%)$ son de zona rural y $39(52,00 \%)$ son de zona urbana. $6(8,10 \%)$ son de Albacete, $16(21,60 \%)$ de Ciudad Real, $38(51,40 \%)$ de Cuenca, 8 $(10,80 \%)$ de Toledo, $1(1,30 \%)$ de Guadalajara y los $6(6,80 \%)$ participantes restantes pertenecen a otras Comunidades Autónomas.

\section{Resultados}

Ante la pregunta sobre si tenían información de las salidas profesionales al inicio de la carrera, el 70,70\% informó que sí tenía información, frente al 29,30\%.

El 50,90\% informó que había recibido información y asesoramiento durante la carrera y el $49,10 \%$ al final de la misma. Destacamos que, casi un 30\%, manifiesta no poseer información acerca de la proyección laboral de sus estudios y que una buena parte la recibe al final de la carrera. No se pidieron datos acerca de quién les informó y, por tanto, desconocemos el nivel de cualificación de estas informaciones. Podemos conjeturar, sin embargo, que las fuentes habrán sido muy variadas: amigos, profesionales, padres, compañeros de carrera, etc.

En la actualidad, trabajan $53(69,70 \%)$ de los participantes y $51(81,00 \%)$ manifiesta que el trabajo que están desempeñando mantiene una relación directa con los estudios realizados. Además, $50(79,40 \%)$ indica que es necesario el título académico cursado para poder ocupar su puesto de trabajo. Con relación a los participantes que están desempeñando un puesto de trabajo, el $70,20 \%$ tienen un contrato temporal y el $29,80 \%$ un contrato fijo. Sin embargo, entre los participantes que actualmente no están trabajando, el 70,40\% ha trabaja- 
do durante menos de un año, el 18,50\% durante más de un año y, finalmente, el 11,10\% durante más de dos años.

Respecto del tiempo transcurrido entre la finalización de los estudios y el desempeño de un puesto de trabajo, 9 participantes $(14,50 \%)$ ocuparon una actividad profesional al mes, $10(16,10 \%)$ a los dos meses, $18(28,60 \%)$ a los doce meses, $6(9,70 \%)$ a los 24 meses, y el resto en diferentes periodos temporales. La gran mayoría manifiesta sentirse satisfecho con el trabajo desempeñado en alguna ocasión $(74,00 \%)$, frente al $21,90 \%$ que indica a veces y el $4,10 \%$ que no está satisfecho. Este último resultado puede ser que esté relacionado con los que nunca han trabajado, o han realizado dicha función en condiciones muy precarias.

Entre los participantes que en alguna ocasión han desempeñado alguna actividad laboral, el $60,60 \%$ la ha realizado en su propia provincia, el $19,70 \%$ en su región y el $19,70 \%$ restante en otras CC.AA.

Otra de las cuestiones que se plantearon en la encuesta fue la relativa a la formación recibida tras finalizar la carrera (tabla 2). Los datos indican que una mayor formación incrementa la probabilidad de obtener un trabajo. Por ejemplo, entre los $9(11,80 \%)$ participantes que han cursado un master, $7(77,80 \%)$ están trabajando en la actualidad, frente al $69,70 \%$ de la totalidad de participantes.

TABLA 2: Formación recibida tras finalizar los estudios reglados

\begin{tabular}{|l|c|}
\hline \multicolumn{1}{|c|}{ Tipo de formación } & $\%$ \\
\hline Master de especialización y postgrado & 11,80 \\
\hline Cursos cortos de especialización & 85,50 \\
\hline Cursos de idiomas & 10,50 \\
\hline Cursos de habilidades sociales & 26,30 \\
\hline Actualización en general & 22,40 \\
\hline Cursos relacionados con otros estudios & 27,60 \\
\hline Trabajos de investigación y doctorado & 6,60 \\
\hline Otros estudios & 25,00 \\
\hline
\end{tabular}

$\%=$ Porcentaje.

Tras la finalización de los estudios, el 78,70\% indica que no mantiene ningún contacto con la Universidad, frente al $20,00 \%$ que manifiesta tener algún contacto y, únicamente, el $1,30 \%$ mantiene un gran contacto. Es más, el 94,60\% manifiesta que la Universidad no le ha facilitado ninguna ayuda posterior a la finalización de los estudios para encontrar un trabajo.

No obstante, ante la pregunta de "si tuviera la oportunidad de volver a comenzar los estudios universitarios realizaría el mismo tipo de estudios", el 90,10\% señala que "sí".

Finalmente, en función de la carrera analizada, cabe destacar una serie de cuestiones (tabla 3). Los participantes que están cursando Trabajo Social, en mayor medida proceden de la zona rural. Al inicio de la carrera, el alumnado de enfermería tenía una elevada información sobre la misma (82,35\%), frente al de Bellas Artes (38,50\%). Además, en esta últi- 
ma carrera, no se considera necesario el título para desempeñar un puesto de trabajo. No obstante, el $100 \%$ volvería a cursar los mismos estudios.

Tabla 3. Información sobre las tres carreras

\begin{tabular}{|l|c|c|c|c|c|}
\hline \multicolumn{1}{|c|}{ Carrera } & A & B & C & D & E \\
\hline Trabajo Social & 57,14 & 71,42 & 78,60 & 65,20 & 81,48 \\
\hline Bellas Artes & 41,70 & 38,50 & 69,20 & 50,00 & 100,00 \\
\hline Enfermería & 44,10 & 82,35 & 61,76 & 100,00 & 93,75 \\
\hline
\end{tabular}

$\mathrm{A}=$ Zona rural; $\mathrm{B}=$ Tener información al inicio de la carrera; $\mathrm{C}=$ Trabajan en el momento actual; $\mathrm{D}=$ Necesario el título para el trabajo que se desempeña; $\mathrm{E}=\mathrm{Vol}$ ver a cursar la misma carrera.

\section{Conclusiones e implicación práctica}

\section{Aproximación a un servicio de orientación}

Incluiría los distintos ámbitos necesarios para una orientación completa de los universitarios, es decir, aprendizaje, atención a la diversidad, orientación académica y profesional y prevención y desarrollo personal. Para un buen funcionamiento, debe haber interacción constante entre los espacios enumerados; por ejemplo, un programa de estrategias para la búsqueda de empleo debe tener en cuenta también la diversidad de cada sujeto, sus aspiraciones personales y su contexto concreto. En este sentido, tras una intervención global y "dirigida a todos", será necesario concretar la orientación "para cada uno", en función de sus peculiaridades personales.

Al mismo tiempo, un servicio de estas características podría incluir los distintos modelos con los que actualmente se trabaja en orientación: counseling o intervención directa, programas y asesoramiento o consulta. El counseling terapéutico puede utilizarse en el apoyo directo a aquellas situaciones personales manifiestamente necesitadas de ayuda, aunque la entrevista deba ser también el espacio ordinario de orientación. Los programas de diversa índole, planteados siempre en función de necesidades y bien enraizados en el contexto, constituyen una vía de intervención muy utilizada para abordar racionalmente distintas realidades universitarias: prevención de la drogadicción, técnicas de aprendizaje, toma de decisiones, etc. Por otra parte, cuando la demanda de ayuda procede de un colectivo, planificar un asesoramiento puede ser lo más pertinente; por ejemplo, si un grupo de tutores solicitan formación para orientar a sus estudiantes acerca de las salidas laborales. No cabe duda que, en ocasiones, el asesoramiento puede producirse en la ayuda al desarrollo de programas.

\section{Referencias bibliográficas}

Bayot, A.; del Rincón, B.; Racionero, C. F. y González, S. (2000). "Resultados preliminares de un Servicio de Orientación Universitaria en Castilla-La Mancha”. En Salmerón, H. y López, V. L. (coords.), Orientación educativa en las universidades. Granada: Grupo Editorial Universitario. 
Del Rincón, B.; Bayot, A.; García, A. y Argudo, S. (2000). "El servicio de orientación universitaria de Cuenca: justificación y trayectoria". En Salmerón, H. y López, V. L. (coords.), Orientación educativa en las universidades. Granada: Grupo Editorial Universitario, pp. 311-320.

Martínez Aldanondo, J. (2002). "Los 2 problemas de la Universidad: qué se enseña y cómo se enseña". En Actas de la $2^{a}$ Conferencia Internacional de la educación y de la formación basada en las nuevas tecnologías Online Educa Barcelona. Barcelona: ICWE GmbH, pp. 31-34.

Vázquez, G.; Colom, A. y Sarramona, J. (1998). "Evaluación de la Universidad. Criterios de calidad". Teoría de la educación, 10, pp. 55-58.

Vieira, M. J. y Vidal, J. (2006). "Tendencias de la educación superior europea e implicaciones para la orientación universitaria”. Revista Española de Orientación y Psicopedagogía, vol. 17, 1, ${ }^{\mathrm{er}}$ semestre, pp. 75-97.

\section{ANEXO \\ Cuestionario a egresados de la Universidad de Castilla-La Mancha}

Nota aclaratoria:

Este cuestionario forma parte de un trabajo de investigación de la Facultad de Ciencias de la Educación y Humanidades. Tu colaboración es muy importante y puede resultar beneficiosa para el futuro.

El cuestionario está dirigido a alumno/as que acabaron sus estudios en los cursos $1994 / 95$ y $1996 / 97$.

Carrera que has estudiado:

Edad: Sexo: $\square$ Hombre $\square$ Mujer

Año de comienzo de estudios:

Provincia de Procedencia: $\square$ AB $\square$ CR $\square$ CU $\square$ TO Origen: $\square$ Rural $\square$ Urbano

1. Al inicio de la carrera ¿Sabías de las salidas profesionales de la misma? $\square$ Sí $\square$ NO

2. ¿Te ofrecieron información y asesoramiento sobre las salidas profesionales?

$\square$ Durante la carrera $\square$ Al final de la carrera (incluir grado)

3. ¿Estás trabajando actualmente? $\square$ Sí $\square$ NO

4. Tu trabajo ¿tiene relación directa con lo que estudiaste? $\square$ Sí $\square$ NO

5. El título académico que tienes ¿es necesario para el trabajo que ocupas? $\square$ Sí $\square$ NO

6. Si tienes trabajo, el contrato ¿es fijo o temporal? $\square$ FIJO $\square$ TEMPORAL

7. Si estás desocupado/a ¿has trabajado alguna vez desde que acabaste los estudios? $\square$ NUNCA $\square$ - DE UN AÑO $\square$ + DE UN AÑO $\square$ + DE DOS AÑOS

8. Si has trabajado (en general) o trabajas ¿cuánto tiempo tardaste en tener un primer contrato desde que acabaste tus estudios?

9. Si has trabajado alguna vez ¿te has sentido satisfecho/a con el trabajo que hacías o que haces? $\square$ Sí $\square$ NO $\square$ A VECES 
10. Si has trabajado o trabajas ¿dónde lo haces?

$\square$ EN MI PROVINCIA $\square$ EN LA REGIÓN $\square$ FUERA DE LA REGIÓN

11. Desde que acabaste la carrera ¿cómo has mejorado y complementado tu curriculum una vez acabados tus estudios? (Contesta con X los items que consideres oportuno)

$\square$ Master de Especialización y postgrados.

$\square$ Cursos cortos de especialización.

$\square$ Estudios de idiomas (especifica).

$\square$ Cursos de habilidades sociales y personales.

$\square$ Actualización genérica.

$\square$ Cursos relacionados con otros estudios.

$\square$ Trabajos de investigación y doctorado.

$\square$ Otros (especificar cuales).

12. ¿Sigues manteniendo algún contacto con la Escuela o Facultad dónde estudiaste?

$\square$ MUCHA $\square$ ALGUNA $\square$ NINGUNA

13. Si mantienes algún tipo de contacto ¿Puedes especificar de que tipo?

14. La Universidad además de facilitarte un título ¿te ha ayudado posteriormente a encontrar empleo? $\square$ SI $\square$ NO

15. Desde tu experiencia ¿qué aconsejarías a los recientes titulados de cara a encontrar trabajo?

16. Si tuvieras la oportunidad de volver a comenzar estudios universitarios desde secundaria ¿realizarías el mismo tipo de estudios?

$\square$ No, porque no encuentro salidas laborales.

$\square$ No, porque no estoy satisfecho.

$\square$ Sí.

Fecha de recepción: 28-04-2006

Fecha de revisión: 21-02-2008

Fecha de aceptación: 22-05-08 